\title{
Efectos de un videojuego de simulación sobre tendencias altruistas en un contexto de educación abierta
}

Effects of a Simulation Videogame on Altruistic Tendencies in an Open Education Context

Efeitos de um videogame de simulação sobre tendências altruístas em um contexto de educação aberta

Gabriela Garbanzo-Rodríguez* (iD o000-0001-8303-7236

Cristina Rivera-Villareal ** (iD 000-0003-2455-4043

Vanessa Smith-Castro*** (iD o000-0001-6348-4223

Rolando Pérez-Sánchez ${ }^{* * *}$ (iD $0000-0001-6321-2543$

Artículo de investigación

Revista Colombiana de Educación, N.7 73. Segundo semestre de 2017, Bogotá, Colombia.

doi: $10.17227 / 01203916.73$ rce39.56

Para citar este artículo: Garbanzo-Rodríguez, G., Pérez-Sánchez, R., Rivera-Villareal, C., y Smith-Castro, V. (2017). Efectos de un videojuego de simulación sobre tendencias altruistas en un contexto de educación abierta. Revista Colombiana de Educación, (73), 41-58.

(c) $\underset{\mathrm{BY}}{(1)(8)}$

Recibido: 12/12/2015

Evaluado: 12/02/2017

* Instituto de Investigaciones Psicológicas, Universidad de Costa Rica. Correo electrónico: gabrielagarbanzo(a)gmail.com

** Instituto de Investigaciones Psicológicas, Universidad de Costa Rica. Correo electrónico: cristinariverav@gmail.com

*** Instituto de Investigaciones Psicológicas, Universidad de Costa Rica. Correo electrónico: vanessa.smith@ucr.ac.cr

**** Instituto de Investigaciones Psicológicas, Universidad de Costa Rica. Correo electrónico: rolarez@gmail.com 


\section{Resumen}

La presente investigación se dirigió a analizar el efecto del uso de un videojuego de simulación para el entretenimiento en la tendencia altruista de una muestra no probabilística de 56 personas con una edad promedio de 32,63 años, participantes de un programa comunal de capacitación en el uso de la computadora. Mediante un diseño cuasiexperimental con pretest y pos-test se expuso a los participantes al uso de un videojuego de simulación en cuatro condiciones (juego solo, juego en parejas, juego con instrucción, juego sin instrucción). Se observó un aumento significativo en las puntuaciones de la medida de altruismo si el juego se acompañaba de una instrucción pedagógica. En su ausencia, las medidas de altruismo descendieron significativamente. No se mostraron cambios en la tendencia altruista asociados al uso del videojuego en parejas o en solitario. En general, el estudio apoya la idea de que el uso de un videojuego con contenido prosocial se encuentra vinculado con las respuestas prosociales de los usuarios.

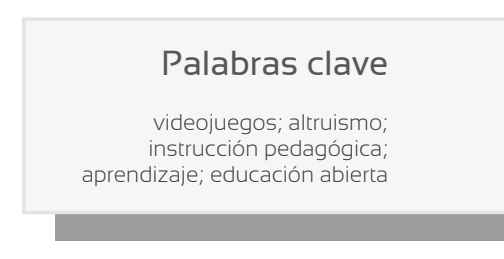

Abstract

The purpose of this investigation was to analyze the effects of using a simulation videogame for entertainment in the altruistic tendencies of a non-probabilistic sample of 56 people with an average age of 32.63 years who participated in a community training program in computer use. Through a quasi-experimental design with a pre-test and a posttest, participants were exposed to the use of a simulation videogame with four modes (single player, two players, playing with instructions, and playing without instructions). We observed a significant increase in altruism measuring scores when the game included a pedagogical instruction. When there were no instructions, the altruism scores dropped significantly. There were no changes in the altruistic tendencies associated with playing in single mode or in pairs. Overall, the study supports the idea that the use of videogames with prosocial content is linked to the prosocial responses of users.

\section{Resumo}

A presente pesquisa visa analisar o efeito do uso de um videogame de simulação para o entretenimento na tendência altruísta de uma mostra não probabilística de 56 pessoas em uma idade média de 32 a 63 anos, participantes de um programa comunal de capa. citação no uso do computador. Por meio de um procedimento quaseexperimental com pré-teste e pós-teste, os participantes interatuaram com um videogame de simulação em quatro condições (jogo solo, jogo em duplas, jogo com instrução, jogo sem instrução). Evidenciou-se um incremento significativo nas pontuações da medida de altruísmo se o jogo era acompanhado de uma instrução pedagógica. Na ausência desta, as medidas de altruísmo descenderam significativamente. Não se evidenciaram mudanças na tendência altruísta associadas ao uso do videogame em duplas ou em solitário. Em geral, o estudo apoia a ideia de que o uso de um videogame com conteúdo pro-social está vinculado com as respostas pro-sociais dos usuários.

\section{Palavras chave}

videogames; altruismo; instrução pedagógica; aprendizagem; educação aberta 


\section{Introducción}

Actualmente, las herramientas digitales como los videojuegos de entretenimiento se han convertido en un importante medio de inserción a la cultura de la informática en niños, niñas, adolescentes y adultos (Granic, Lobel y Engels, 2014). Sin embargo, su aplicación en programas de enseñanza para adultos solo ha sido considerada de manera incipiente. Hasta ahora los resultados se han orientado a su uso en los demás grupos.

A nivel educativo se han encontrado resultados positivos asociados a la introducción de videojuegos en contextos de aprendizaje al compararlos con métodos de enseñanza tradicional, y al uso de juegos de video educativos en población infantil y adolescente (Boyle, Hainey, Connolly, Gray, Earp, Ott et al., 2016). Los resultados apuntan a que los videojuegos incrementaron los procesos de atención, acción y recuerdo, y además promovieron habilidades de resolución de problemas, espaciales y mejoraron el rendimiento en materias específicas.

Otro ejemplo de este tipo de investigación es el trabajo de Panoutsopoulos y Sampson (2012), quienes indagaron el efecto de un videojuego comercial en el aprendizaje académico en colegiales. Un resultado destacado fue una mejor consecución de los objetivos educativos en la enseñanza de la matemática con el uso de esta herramienta.

Considerando el vacío existente en indagaciones con población adulta y partiendo de la necesidad de innovar en las estrategias de enseñanza, incorporando alternativas como los videojuegos, con esta investigación se pretendió evaluar la aplicación de un videojuego de simulación en un ambiente de aprendizaje para adultos. Específicamente, el estudio evaluó cambios en el razonamiento prosocial luego de introducir el videojuego de simulación, considerando varias condiciones: la indicación o ausencia de indicación del objetivo pedagógico y el trabajo individual o en parejas.

Los juegos de simulación también son llamados de estrategia, y su objetivo es la adquisición o el manejo de recursos en un ambiente o sociedad virtual para la consecución de un objetivo que se alcanza de manera escalonada o por niveles (Smith, 2006). En este contexto, pueden mantener relación de cooperación o competencia con otras sociedades para obtener los recursos que se necesiten en un momento dado. Con los materiales o servicios adquiridos la persona puede construir o desarrollar su sociedad. El juego demanda un tipo de actividad cognitiva asociada con la resolución de problemas que tiene en cuenta diferentes variables, a la vez que se interactúa para avanzar o retroceder en la construcción de la sociedad virtual. 
El objetivo de los juegos de simulación es recrear acontecimientos que suceden en la vida real. Para este estudio se recurrió a un tipo específico de juego de simulación que se dirige a crear, desarrollar y mantener una ciudad, por lo que resulta una herramienta relevante para medir efectos en la orientación prosocial. La escogencia de este tipo de juego se fundamenta precisamente en el hecho de que el estudio se llevó a cabo en un programa de educación abierta, orientado específicamente al desarrollo de la comunidad en la que se inscribe.

El aprendizaje se considera aquí como un proceso activo, que depende de la asimilación de contenidos mediante la relación con informaciones previas del individuo (Yaran, 2014). Ausubel, Novak y Hanesian (1983) proponen que el aprendizaje se genera una vez que la nueva información se relaciona con los conocimientos y con las experiencias previas que posee la persona en su estructura cognitiva. A través de reacomodos de esta se incorporan los nuevos datos, lo que facilita una conservación más duradera de los contenidos, propiciada por la motivación que tenga la persona para aprender (Yaran, 2014).

La teoría del aprendizaje significativo expresa, con relación a la población adulta, que el aprendizaje por recepción adquiere mayor relevancia en sus formas verbales, pues sugiere un proceso de asimilación y un nivel mayor de madurez cognoscitiva (Ruiz, 2001). Según Ruiz (2001), el repertorio emocional y el dominio del razonamiento más formal y abstracto en la edad adulta constituyen una relación lateral en la cual se incrementa la flexibilidad de los procesos de afrontamiento y defensa ante situaciones conflictivas. Asimismo, el adulto tiene su propio razonamiento (sus propios criterios y experiencias) y su lenguaje emocional es más extendido.

La interacción con el medio y las situaciones asociadas con el aprendizaje facilitan la efectividad de la instrucción. Aushburn (2006) señala que se debe atraer la atención del aprendiz, informarle sobre los objetivos y asegurarse de que los comprende, estimular conocimientos previos, proveer material atractivo orientado a la consecución del aprendizaje (en donde se facilite el alcance de los objetivos) y brindar una retroalimentación inmediata de su proceso, que le permita valorar su aprendizaje.

El aprendizaje concreto que se pretendió abordar en este estudio es el que tiene que ver con la orientación prosocial. Esta puede vislumbrarse en aquellas actitudes asociadas a conductas sociales de efectos positivos, que tienen como propósito ayudar a otros (Keltner, Kogan, Piff y Saturn, 2014; Simpson y Willer, 2015). La conducta prosocial es un constructo compuesto por sistemas y capacidades individuales tanto cognitivos como afectivos que se influyen mutuamente (Moñivas, 1996). 
Para Keltner et al. (2014), con la conducta prosocial se asocian variables como la experiencia, las expectativas, las recompensas, los factores disposicionales y los factores situacionales (como el estado de ánimo). La dimensión cognitiva de estas conductas se conoce como razonamiento moral prosocial y determina la calidad de las conductas prosociales (Eisenberg, Hofer, Sulik y Liew, 2014). El razonamiento moral prosocial se apoya en dilemas relacionados con el conflicto entre los deseos o necesidades de un individuo frente a los de otras personas, en un contexto de mínimas consecuencias punitivas.

La orientación prosocial altruista se define como aquellas actitudes vinculadas al respaldo de conductas voluntarias de ayuda, motivada por la visión de las necesidades y el bienestar de los demás, e inducida por la empatía y la internalización de normas y principios relacionados con la ayuda a otros. En las conductas altruistas se excluye la voluntad de obtener recompensas a corto o largo plazo (Feigin, Owens y Goodyear-Smith, 2014).

La empatía es uno de los elementos que motiva a las conductas altruistas. Se define como una reacción emocional que concuerda con un estado emotivo de quien ayuda, basado en la percepción y la comprensión del estado emocional de quien requiere la ayuda, es decir que se sucede una toma de perspectiva del punto de vista del otro (Batson, 2009). Así, se ha evidenciado que especialmente cuando se trata de sujetos adultos, las personas que muestran alta empatía realizan más conductas altruistas que aquellas con baja empatía (Batson, 2011).

El otro factor influyente en la motivación hacia este tipo de conductas son las normas y los principios concernientes a la acción de brindar ayuda. De acuerdo con diferentes investigaciones (Carlo y Randall, 2002; Feigin et al., 2014), las personas que demuestran altos niveles de razonamiento moral y realizan conductas consistentes con este han adquirido un sentido de responsabilidad conforme a sus principios. Por tanto, la conducta prosocial altruista está asociada con una responsabilidad social internalizada (Carlo, Christ, Laible y Gulseven, 2015).

La investigación sobre videojuegos prosociales y su asociación con la conducta prosocial se mantiene como un tema poco abordado. Los estudios existentes muestran, sin embargo, resultados relevantes. Tanto Greitmeyer y Oswald (2010), como Greitmeyer, Oswald Brauer (2010) encontraron que el uso de juegos prosociales está asociado positivamente con la evocación de afectos prosociales y negativamente con la presencia de afectos antisociales. Por su parte, Greitmeyer y Mügge (2014) Ilevaron a cabo un metaanálisis con 98 artículos que indagaban acerca de la asociación entre videojuegos prosociales y respuestas agresivas y prosociales. Encontraron que la participación en videojuegos prosociales disminuye la 
presencia de conductas, pensamientos y emociones agresivas, y aumenta la de conductas, emociones y pensamientos prosociales. Estos resultados se presentan de forma consistente tanto en estudios experimentales, correlacionales y longitudinales como en aquellos que evalúan efectos a corto y largo plazo. En esta misma línea, Harrington y O'Connel (2016) encontraron que los videojuegos prosociales se asociaron con el mantenimiento de relaciones afectivas positivas, la tendencia a cooperar y compartir, así como puntajes altos en empatía. Estos resultados se encontraron con independencia de la condición socioeconómica, el sexo, la frecuencia de uso o la participación en videojuegos violentos.

El aporte particular de la presente investigación consiste en la evaluación de las posibilidades educativas de un videojuego comercial de simulación dirigido al entretenimiento, y está guiado por la siguiente pregunta de investigación básica: ¿Cuál es el efecto del uso de un videojuego de simulación de entretenimiento en la orientación prosocial en adultos participantes de un programa comunal de capacitación en herramientas básicas de computación?

La investigación busca contrastar empíricamente las siguientes hipótesis:

» H1: El uso del videojuego de simulación de entretenimiento con instrucción del objetivo pedagógico acrecienta la orientación prosocial en los participantes.

» $\mathrm{H} 2$ : El uso del videojuego de simulación de entretenimiento en parejas acrecienta la orientación prosocial en los participantes.

A continuación se presentan los procedimientos para contrastar estas hipótesis.

\section{Método}

Se llevó a cabo un diseño cuasiexperimental mixto con medición pre- y pos-test, y dos factores entre sujetos: tipo de instrucción (con o sin indicación del objetivo pedagógico) y contexto de uso (individual o en parejas).

\section{Participantes}

Según un análisis de poder prospectivo, para un efecto de mediano tamaño ( $d$ de Cohen $=, 50)$, una probabilidad de cometer el error alfa del $5 \%$ y una probabilidad de cometer el error beta del $20 \%(\alpha=, 05$ y $\beta=, 20)$, el número mínimo de participantes por condición debía ser de al menos 14 personas (un total de 56 participantes para las 4 condiciones entre sujetos), tamaño muestral requerido para alcanzar un poder de ,80 (Clark-Carter, 2004). 
Así, participaron en total 56 individuos de ambos sexos, cuya edad promedio fue de 32,63 años ( $D E=15,65)$. De la muestra, el $75 \%$ correspondió a mujeres $(n=42)$ y el $25 \%$ fueron hombres $(n=14)$.

\section{Instrumentos}

El cuestionario suministrado como pre- y pos-test contó con un encabezado que informaba sobre las particularidades del estudio, y el consentimiento informado que seguía los lineamientos del Comité de Ética de la Universidad Costa Rica. El instrumento incluyó el módulo de variables sociodemográficas, edad, sexo, nivel educativo, oficio, estado civil, número de hijos y la medida de conducta prosocial.

La medición de la conducta prosocial se llevó a cabo mediante una adaptación de la escala de autorreporte Prosocial Tendencies Measure (PTM) de Carlo y Randall (2002), que indaga sobre la preferencia por comportarse prosocialmente. La escala original consta de 22 ítems ( $\square$ de Cronbach $=, 89$ ) distribuidos en 6 subescalas relacionadas con los tipos de conducta prosocial: altruista, anónima, pública, emergencia, emotiva y por complacencia. Algunos ejemplos de los reactivos que componen la escala son: "tiendo a ayudar más a los necesitados cuando ellos no saben quién los ayudó", "acostumbro ayudar a mis vecinos cuando están en circunstancias muy conmovedoras", "me inclino a ayudar a organizaciones de mi comunidad que reconozcan mi trabajo". Cada uno de los reactivos se contestaba mediante una escala tipo Likert de 1 (No me describe) a 5 (Me describe totalmente).

La dimensión altruista representó con mayor consistencia el ámbito de la conducta prosocial contenido en el videojuego de simulación utilizado en el estudio. En consecuencia únicamente se tomó en cuenta la subescala de altruismo $(\alpha$ de Cronbach $=, 81$ ) como la dimensión principal para la valoración de la conducta prosocial. A cada participante se le calculó el índice de tendencias altruistas a partir del promedio de las respuestas a los ítems que componen esta subescala, en donde altas puntuaciones indican altos niveles de altruismo.

\section{Estímulos}

Para indagación del efecto del uso de un videojuego de entretenimiento en la conducta prosocial se utilizó el dispositivo SimCity 3000 (Maxis), ya que sus contenidos resultan significativos para el aprendizaje al exponer al usuario a la ejecución de actividades relacionadas con situaciones de la vida cotidiana de una comunidad. 
El juego de simulación SimCity 3000 fue desarrollado por el estudio Maxis y forma parte de la categoría de videojuegos de simulación por computadora. Estos son sistemas referidos a la construcción de ambientes digitales, donde el usuario interactúa y construye relaciones sociales y modelos de colaboración debido a la sensación de que temporalmente se pueden trascender las limitaciones sociales y físicas (Smith, 2006).

La misión del juego es construir, desarrollar y administrar una ciudad con un amplio sentido del urbanismo, con sus correspondientes servicios públicos, previendo un adecuado desarrollo económico, social y ambiental, mediante la acción del usuario concebido como el alcalde de la ciudad.

A diferencia de otros juegos de simulación o estrategia, en donde se introducen contenidos bélicos o de competencia entre comunidades o sociedades, en este la actividad se centra en las habilidades del jugador para el desarrollo y la administración de la comunidad. Esta característica facilita la evocación de pensamientos altruistas, ya que del jugador dependerá precisamente de que la ciudad se mantenga y mejore en el tiempo. De allí su elección para probar las hipótesis del estudio.

\section{Procedimiento}

La recopilación de los datos se realizó por medio de un cuestionario suministrado en dos momentos, antes y después de la situación experimental, en tres etapas. En la fase I se aplicó un pre-test que incluyó un cuestionario sobre datos sociodemográficos y la escala PTM. Enseguida, en la fase II se aplicó el diseño pedagógico, que consistió en una sesión inicial de una hora de capacitación sobre el uso del juego SimCity 3000 a los participantes. El equipo de investigación asistió a la muestra en dudas relacionadas con los comandos del juego. Posteriormente, se aplicó el estímulo experimental en cuatro sesiones de juego de una hora para los cuatro grupos (individual/ parejas y con/sin instrucción del objetivo pedagógico). A los participantes en la condición de instrucción se les indicó lo siguiente: "El objetivo del juego es ser el alcalde de una ciudad que hay que construir y desarrollar tomando en cuenta las necesidades de los habitantes y de la comunidad", mientras que los participantes en la condición sin instrucción recibieron solamente la siguiente consigna: "El objetivo del juego es construir una ciudad". Cada consigna se trabajó en solitario y en parejas. Finalmente, en la fase III se administró el pos-test, esta vez únicamente con la escala PTM para la medición de la conducta prosocial. 


\section{Estrategia de análisis}

Los análisis descriptivos preliminares incluyeron pruebas $t$ para muestras independientes, pruebas de chi-cuadrada y correlaciones bivariadas con el fin de estimar la comparabilidad de los participantes en las condiciones experimentales y la incorporación de potenciales covariables. Para contrastar las hipótesis se ejecutó un análisis de varianza (Anova) mixto con comparación intra-sujetos (pretest frente a pos-test) y entre-sujetos (condiciones experimentales), seguido de una serie de pruebas $t$ para grupos independientes y apareados.

\section{Resultados}

\section{Análisis preliminares}

Debido a que se trabajó con grupos intactos, primero se estimaron las potenciales diferencias de edad y escolaridad entre los participantes de las distintas condiciones. Asimismo se analizó la distribución por sexo en cada grupo y se estimaron las correlaciones entre la medida de altruismo y las características sociodemográficas de los participantes.

No se encontraron diferencias estadísticamente significativas en la edad de los grupos según el factor instrucción $\left(t_{52}=, 72, p=, 47\right)$. Tampoco teniendo en cuenta el factor de contexto de uso $\left(t_{52}=1,58, p=, 12\right)$. De la misma manera, no se encontraron diferencias en el nivel educativo de los grupos según el tipo de instrucción, $\left(t_{52}=, 58, p=, 57\right)$, ni entre los grupos del factor de contexto de uso $\left(t_{47}=1,87, p=, 07\right)$. Las pruebas de chi cuadrada indicaron que la distribución del sexo es similar tanto en los participantes agrupados según el factor del tipo de instrucción $\left(\chi_{(1)}^{2}=2,90\right.$, $p=, 09)$ como en los participantes agrupados en el factor de contexto de uso $\left(\chi_{(1)}^{2}=, 21, p=, 64\right)$.

En general, los análisis preliminares indicaron que los grupos eran relativamente equivalentes en términos del sexo, la edad y el grado de escolaridad de sus participantes. Esto es, que las condiciones no se confundieron con las variables sociodemográficas de los participantes en cada uno de los niveles de las mismas. Por otro lado, las correlaciones entre el sexo, la edad y la escolaridad y las variables dependientes (puntuaciones del altruismo) revelaron que ninguna de las variables demográficas covariaba con ninguna de las mediciones de la variable dependiente (todas las $r_{\mathrm{s}}<, 23$, todas las $\left.p_{\mathrm{s}}>, 11\right)$. Lo anterior justificó la ausencia de covariables en los análisis principales. 


\section{Efecto del tipo de instrucción y el contexto de uso del videojuego sobre el altruismo}

Tal y como se mencionó, la contrastación de las hipótesis se llevó a cabo mediante un análisis de varianza (Anova) mixto 2 (tiempo) $\times 2$ (tipo e instrucción) $\times 2$ (contexto de uso), con el tiempo de medición (pretest frente a pos-test) como factor intra-sujetos y las condiciones experimentales como factores entre-sujetos.

El Anova no evidenció un efecto significativo del tiempo (pretest y pos-test) en sí mismo $F_{(1,50)}=, 21, p=, 65, \eta^{2}=, 004$, pero sí una interacción entre el tiempo y el tipo de instrucción, $F_{(1,50)}=10,58, p<, 002, \eta^{2}=, 18$ ), lo que indica que las diferencias en los puntajes de altruismo antes y después de la intervención dependían del tipo de instrucción que se les dio a los participantes. La interacción entre el tiempo de medición y el contexto de uso no resultó significativa, $F_{(1,50)}=, 28, p=, 60, \eta^{2}=, 005$; tampoco la interacción de tres vías entre el tiempo de medición, el tipo de instrucción y el contexto de uso del videojuego, $F_{(1,50)}=, 45, p=, 51, \eta^{2}=, 009$. Los resultados de este análisis se presentan en la tabla 1.

Tabla 1. Resultados del análisis de varianza de la tendencia prosocial

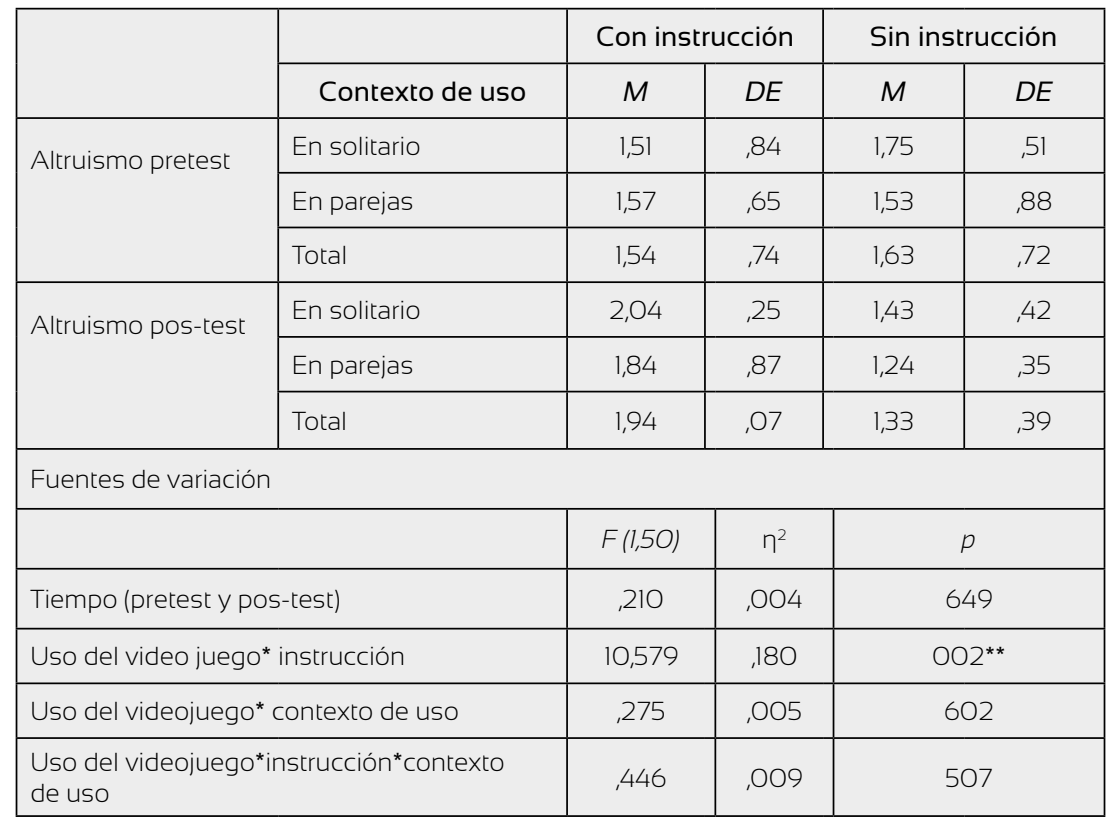

$M=$ Media, $D E=$ Desviación estándar, $F=$ Razón de Fisher, $\mathrm{gl}=$ grados de libertad. ${ }^{* *} p<, 01$. 
Para examinar la interacción entre los distintos grupos se compararon las puntuaciones medias del pretest del grupo sin instrucción con las del grupo con instrucción, mediante pruebas $t$ para muestras independientes. De igual manera se procedió con los puntajes del pos-test. Además se comparó el cambio del altruismo entre la primera medición y la segunda para cada grupo por separado, mediante pruebas $t$ para muestras apareadas. Las medias relevantes para este análisis se encuentran también en la tabla 1.

La prueba $t$ para la comparación de las puntuaciones del altruismo en el pretest indicó que los grupos no se diferenciaban significativamente en esa variable antes de usar los videojuegos $\left(t_{52}=, 44, p=, 662\right)$. Por otro lado, la prueba $t$ para las puntuaciones del altruismo en el pos-test indicó que los grupos si se diferencian significativamente después de la intervención $\left(t_{52}=-2,84, p=, 007\right)$. Los datos señalan que el grupo que recibió instrucciones que indicaban el objetivo pedagógico del juego presentaron niveles de altruismo significativamente mayores $(M=1,94, D E=, 07)$ que aquellos que no las recibieron $(M=1,33, \mathrm{DE}=, 39)$.

Las pruebas $t$ para muestras apareadas indicaron que entre quienes recibieron instrucciones sobre el objetivo pedagógico del juego, aumentaron significativamente la tendencia altruista $\left(t_{27}=-2,21, p=, 036\right)$ de 1.54 $(\mathrm{DE}=, 74)$ a $1,94(\mathrm{DE}=, 07)$; mientras que entre quienes no recibieron instrucciones el altruismo disminuyó significativamente de $\left(t_{25}=2,93, p=, 007\right)$ 1,63 (DE=,72) a 1,33 (DE =,39).

Para el caso del grupo con instrucción, se tomó como fórmula para estimar el tamaño del efecto $d=2 t / \sqrt{ }$ gl (Rosenthal y Rosnow, 1991); el aumento en términos de unidades de desviaciones estándar o $d$ de Cohen fue de, 85 , lo que representa un efecto grande. Para el caso del grupo sin instrucción la disminución en términos de $d$ de Cohen fue de 1,17, lo que representa también un efecto relevante. En la figura 1 se observa el comportamiento de las variaciones en las medias de la tendencia altruista según el tipo de instrucción (con o sin la instrucción del objetivo pedagógico) durante las dos mediciones pre- y pos-test.

En síntesis, los datos indican que la instrucción de los objetivos pedagógicos parece aumentar el altruismo de los participantes, mientras que el jugar sin instrucciones está asociado a una disminución de este. Por otro lado, el jugar solo o en pareja parece no tener efecto alguno en el altruismo. 


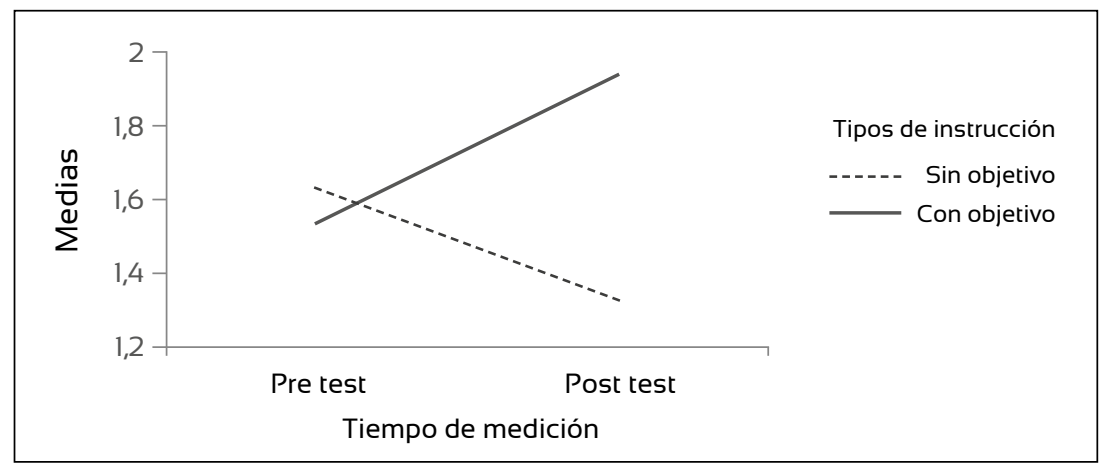

Figura 1. Medias de Altruismo según tipo de instrucción

\section{Discusión y conclusiones}

El presente estudio tuvo como objetivo examinar el potencial impacto de los videojuegos de simulación en las tendencias prosociales. Con respecto a las hipótesis de investigación, se pudo encontrar que el uso del videojuego de simulación de entretenimiento con instrucción del objetivo pedagógico acrecienta la orientación prosocial en los participantes; no obstante, su uso en la modalidad de parejas no acrecienta la orientación prosocial en los participantes en comparación con la modalidad individual.

Se muestra en la presente investigación que el uso de un videojuego con contenido prosocial está relacionado con las respuestas prosociales de los usuarios, en especial con las conductas prosociales de tipo altruista.

En este sentido, y considerando la dimensión cognitiva de la conducta prosocial, participar en un videojuego mediado por una instrucción sobre el objetivo prosocial del mismo parece acrecentar la respuesta altruista de los usuarios. Por lo tanto, en ausencia de dicha instrucción los individuos tienden a disminuir sus respuestas prosociales.

En cuanto a la influencia positiva de la instrucción en la tendencia altruista, se evidencia que la posibilidad de entender las necesidades de los otros con mayor claridad facilita la "toma de perspectiva" (Batson, 2011), que influye en el análisis de las situaciones y circunstancias que rondan las respuestas prosociales. A su vez, esta clarificación que provee la instrucción parece facilitar la activación del razonamiento moral prosocial, lo cual concuerda con Mestre, Frías, Samper y Tur (2002), quienes estiman que los procesos cognitivos relacionados con la edad de los sujetos, así como su formación (diferencias individuales y contextuales), facilitan una madurez en el pensamiento abstracto y la posibilidad de ponerse en el lugar de otros, lo que conduce a respuestas prosociales. 
Por tanto, la combinación de la instrucción (en el uso de un juego de video) junto a un razonamiento más complejo y evolutivamente avanzado puede incidir en el aumento de la orientación prosocial en ese grupo etario. Esto se facilita por una mayor capacidad cognitiva en los adultos que, mediante el pensamiento formal, posibilita la evaluación moral y la toma de perspectivas a partir de la formulación de escenarios hipotéticos o experimentos mentales (Gibbs, 2013). Es decir, el uso de juegos con instrucción parece facilitar el acceso a cogniciones prosociales que derivan en un aumento de la orientación prosocial en adultos; no obstante, futuras investigaciones podrían incluir elementos de la personalidad y la dimensión afectiva que podrían influenciar también la prosocialidad.

Cuando se instruye al jugador, este puede tomar perspectiva de las necesidades de los demás, de forma que pueda actuar en beneficio de otros y prevenir situaciones que los dañen. Este tipo de acciones tienen que ver con aquellas conductas prosociales altruistas en las que el individuo ayuda de forma voluntaria, motivado principalmente por la preocupación hacia las necesidades y el bienestar de otras personas.

El altruismo está inducido por una respuesta simpática y por la internalización de normas y principios consistentes con la ayuda a otros; no así otras conductas prosociales como las de complacencia, o de tipo público, en las cuales la motivación es de tipo extrínseca. En este sentido, quienes recibieron instrucción pedagógica aumentaron su tendencia altruista, pues su juego fue mediado y dirigido hacia un pensamiento más reflexivo en cuanto a las necesidades de los otros y de su entorno. Al respecto, Gibbs (2013) propone que cuando existe una perspectiva socio-moral madura, las relaciones son prosociales y recíprocas, y existe una promoción por parte de los individuos a demandar un sistema social justo y orientado al bien común.

Por tanto quienes no recibieron esta instrucción reflexiva se apegaron a lo que Tapia, Castro y Monestel (2007) Ilaman "una expectativa de conformidad social" (p. 467) asociada a las interacciones socio-morales. Esto se puede trasladar a la tónica de la participación comunitaria en nuestra sociedad, y reproducir en el videojuego, en el cual una figura única (alcalde) es la responsable de todo el acontecer comunitario.

En la investigación de Greitemeyer y Osswald (2010) el uso de un juego de video prosocial por sí mismo produjo respuestas prosociales en los sujetos de estudio, lo cual contrasta con el presente estudio pues el uso de un videojuego sin instrucción provocó una disminución de la tendencia prosocial. Sin embargo, en dicho estudio no existió un test previo a la condición experimental, que permitiera estimar las posibles variaciones en la conducta prosocial después de haber jugado. 
Asimismo, para Chuang y Chen (2009) el simple uso de un videojuego comercial produjo efectos positivos en el aprendizaje, sin que mediara la guía del docente, en este caso, mejorando -en comparación con la enseñanza asistida por computadora- procesos de aprendizaje en escolares, asociados a comprensión, estrategias de resolución de problemas y pensamiento crítico, en un ambiente de simulación. No obstante, la temática de la investigación estaba dirigida hacia procesos cognitivos que no apelaban a dinámicas morales-prosociales como fue la orientación de este estudio.

La reducción de la tendencia prosocial del grupo sin objetivo pedagógico puede entenderse en parte debido a que la ausencia de instrucción encareció la activación de los razonamientos que guían el entendimiento de las situaciones de ayuda incluidas en el videojuego, y que influyen la respuesta altruista.

Cabe mencionar que es necesario estudiar las tendencias prosociales a largo plazo, en especial mediante estudios longitudinales en los cuales se pueda corroborar lo hallado en la presente investigación, y en donde el tiempo de exposición signifique un punto de indagación.

Por otra parte, similar a esta investigación, Panoutsopoulos y Sampson (2012) aplicaron un videojuego comercial en un contexto educativo, y observaron que esta inserción como parte de un diseño pedagógico acompañado de ejercicios en clase puede contribuir al logro de objetivos curriculares, específicamente para la enseñanza de las matemáticas en población infantil. También mediante el uso de ambientes de simulación digital se posibilita un ensayo de los conocimientos adquiridos, lo que favorece un aprendizaje significativo.

Este punto es muy importante para futuras investigaciones o aplicaciones de videojuegos comerciales tanto en entornos de aprendizaje como de entretenimiento, ya que aportar una guía para el desempeño de la actividad lúdica hace que la atención se enfoque en acciones específicas y medie el aprendizaje implícito en el uso del videojuego.

En cuanto a la variable sexo, la investigación de Greitemeyer y Osswald (2010) es coincidente con los resultados de este estudio, pues no se encontraron diferencias por sexo en la respuesta altruista. Este hecho pone a prueba los resultados de la investigación sobre los tipos de conducta prosocial de Carlo y Randall (2002), en los cuales se establece que las mujeres presentan un comportamiento más prosocial que los hombres debido a que poseen razonamientos orientados al cuidado. A diferencia de los resultados del presente estudio, otras investigaciones indican que en mujeres, dichas conductas tienden a ser del tipo altruista, anónimo y emocional con mayor frecuencia, y en los hombres prevalecen las conductas de tipo público y relacionadas con la aprobación de los otros. 
No obstante, según Tapia, Castro y Monestel (2007) las diferencias de género establecidas por Gilligan solo se suceden ante dilemas de la vida real, no al utilizar situaciones hipotéticas, como las que se presentan al usar el videojuego.

La inexistencia de diferencias por sexo concuerdan más bien con el enunciado de Mestre, Delgado, Tur, Diez, Soler y Samper (1999), quienes apuntan que las diferencias por sexo relacionadas con las conductas prosociales disminuyen a edades superiores, en especial en cuanto al razonamiento hedonista y el orientado a la aprobación.

Por otra parte, en cuanto a la hipótesis que enunciaba que el juego en parejas aumentaría la tendencia prosocial, se observó que esta modalidad de juego no incrementa dicha propensión; posiblemente se deba a que por una parte SimCity es un dispositivo de modalidad de juego individual, lo que dificulta el desempeño en parejas, y por otra parte a que en las conductas altruistas, las ayudas que la gente proporciona a otros no dependen de las motivaciones extrínsecas, no están relacionadas con la toma de decisiones basadas en la aprobación u opinión de los otros (pareja, en este caso) como sí sucede en conductas prosociales públicas o por complacencia.

En suma los resultados del presente estudio sugieren que los videojuegos pueden usarse como herramientas pedagógicas con un gran potencial para promover aprendizajes significativos.

\section{Referencias}

Aushburn, E. (2006). It's TIME. Technology integrated into meaningful learning experiences. En E. Aushburn y R. Floden (eds.). Meaningful learning using technology: What educators need to know and do (pp. 1-7). Nueva York: Teachers College Press.

Ausubel, D.; Novak, J. y Hanesian, H. (1983). Psicología educativa: un punto de vista cognoscitivo (2. ${ }^{\text {a }}$ ed.) México: Trillas.

Batson C. D. (2009). These things called empathy: Eight related but distinct phenomena. En Decety J., Ickes W. (eds.), The social neuroscience of empathy (pp. 3-16). Cambridge, MA: MIT Press.

Batson, C. D. (2011). Altruism in humans. Nueva York: Oxford University Press.

Boyle, E. A.; Hainey, T.; Connolly, T. M.; Gray, G.; Earp, J.; Ott, M., ... y Pereira, J. (2016). An update to the systematic literature review of empirical evidence of the impacts and outcomes of computer games and serious games. Computers and Education, 94(3), 178-192. 
Carlo, G.; Christ, C.; Laible, D. y Gulseven, Z. (2015). An evolving and developing field of study: Prosocial morality from a biological, cultural, and developmental perspective. En T. K. Shackelford y R. D. Hansen (eds.). The evolution of morality (pp. 53-76). Nueva York: Springer.

Carlo, G. y Randall, B. (2002). The development of a measure of prosocial behaviors of late adolescents. Journal of Youth and Adolescence, 31, 31-44.

Chuang, T. y Chen, W. (2009). Effect of computer-based video games on children: An experimental study. Educational Technology \& Society, 12(2), 1-10.

Clark-Carter, D. (2004). Quantitative psychological research: A student's handbook. Nueva York: Psychology Press.

Eisenberg, N.; Hofer, C.; Sulik, M. J. y Liew, J. (2014). The development of prosocial moral reasoning and a prosocial orientation in young adulthood: Concurrent and longitudinal correlates. Developmental Psychology, 50, 58-70.

Feigin, S.; Owens, G. y Goodyear-Smith, F. (2014). Theories of human altruism: A systematic review. Annals of Neuroscience and Psychology, 1(1). Recuperado de http://www.vipoa.org/neuropsychol

Gibbs, J. (2013). Moral development and reality (3. ${ }^{a}$ ed.). Nueva York: Oxford University Press.

Granic, I.; Lobel, A. y Engels, R. (2014). The benefits of playing video games. American Psychologist, 69, 66-78.

Greitemeyer, T. y Mügge, D. (2014). Video games do affect social outcomes: A meta-analytic review of the effects of violent and prosocial video game play. Personality and Social Psychology Bulletin, 40, 578589.

Greitemeyer, T. y Osswald, S. (2010). Effects of prosocial video games on prosocial behavior. Journal of Personality and Social Psychology, 98(2), 211-221.

Greitemeyer, T.; Osswald, S. y Brauer, M. (2010). Playing prosocial video games increases empathy and decreases schadenfreude. Emotion, 10(6), 796-802.

Harrington, B. y O'Connel, M. (2016). Video games as virtual teachers: Prosocial video game use by children and adolescents from different socioeconomic groups is associated with increased empathy and prosocial behaviour. Computers in Human Behavior, 63, 650-658.

Keltner, D.; Kogan, A.; Piff, P. K. y Saturn, S. R. (2014). The sociocultural appraisals, values, and emotions (SAVE) framework of prosociality: Core processes from gene to meme. Annual Review of Psychology, $65,425-460$. 
Mestre, V.; Delgado, E.; Tur, A.; Díez, I.; Soler, J. V. y Samper, P. (1999). El razonamiento prosocial en la infancia y en la adolescencia. Un estudio empírico. En V. Maestre y E. Pérez Delgado (eds.). Psicología moral y crecimiento personal (pp. 259-283). Barcelona: Ariel.

Mestre, V.; Frías, D., Samper, P. y Tur, A. (2002). Adaptación y validación en población española del PROM. Una medida objetiva del razonamiento moral prosocial. Revista Acción Psicológica, 1, 221-232.

Moñivas, A. (1996). La conducta prosocial. Cuadernos de Trabajo Social, 9, 125-142.

Panoutsopoulos, H. y Sampson, D. (2012). A study on exploiting commercial digital games into school context. Educational Technology \& Society, 15(1), 15-27.

Rosenthal, R. y Rosnow, R. L. (1991). Essentials of behavioral research: Methods and data analysis (2. ${ }^{\mathrm{a}}$ ed.). Nueva York: McGraw-Hill.

Ruiz, C. (2001). ¿Cómo aprenden los adultos? Características del pensamiento. Revista de Educación a Distancia, 1, 8-13.

Simpson, B. y Willer, R. (2015). Beyond altruism: Sociological foundations of cooperation and prosocial behavior. Annual Review of Sociology, 43, 42-63.

Smith, B. (2006). The computer (games) people play. En P. Vorderer y J. Bryant (eds.). Playing video games, motives, responses, and consequences (pp. 43-56). Nueva Jersey: Lawrence Erlbaum.

Tapia, N.; Castro, R. y Monestel, N. (2007).El desarrollo sociomoral de adolescentes de Costa Rica según el modelo de Gibbs. Revista Latinoamericana de Psicología, 39(3), 449-471.

Yaran, Y. 2015. Education 3-13: International Journal of Primary, Elementary and Early Years Education, 43(1), 83-94. 